Note

\section{New Non-cyclic Homo-diterpene from the Sting Glands of Bracon hebetor Say (Hymenoptera: Braconidae)}

\author{
Jun-ichi Fukushima, Yasumasa Kuwahara,* \\ Akifumi Yamada and Takahisa SUzuKI
}

Institute of Applied Biochemistry, University of Tsukuba, Tsukuba, Ibaraki 305, Japan

Received September 6, 1989

Bracon hebetor Say is a beneficial parasitoid wasp that attacks phycitinae larvae, a pest of stored products, by injecting a venomous liquid. ${ }^{1)}$ The proteinous nature of the venom has been studied physiologically and pharmacologically, ${ }^{2,3}$ but none of the other components are known. In this paper, a novel homo-diterpene was found in an extract of the sting glands (a complex of the venom gland and Dufor's gland ${ }^{4}$ ), along with $\beta$-springene and $n$-alkanes, and was identified as $E, E$-1 I-ethyl-7,15-dimethyl-3-methylene-hexadeca-1,6,10,14-tetraene (tentatively named as homo- $\beta$-springene).

About 6,000 female glands were dipped in hexane to give the extract, which, after filtration and concentration, gave six major peaks by GLC (CP-Sil 19CB, $0.22 \mathrm{~mm} \times$ $25 \mathrm{~m}$, Chrompack, temperature programmed from $80^{\circ} \mathrm{C}$ to $250 \mathrm{C}$ at $7.5 \mathrm{C} / \mathrm{min})$. Peaks D $(21.69 \mathrm{~min}, 7.0 \%), \mathrm{E}$ $(24.08 \mathrm{~min}, 8.4 \%)$ and $\mathrm{F}(27.23 \mathrm{~min}, 2.9 \%)$ were recovered from an $\mathrm{SiO}_{2}$ column by hexane, peaks $\mathrm{A}(17.27 \mathrm{~min}$, $51.9 \%)$ and $\mathrm{B}(18.16 \mathrm{~min}, 19.5 \%)$ by $1 \%$ ether-hexane, and peak $\mathrm{C}(20.26 \mathrm{~min}, 10.4 \%)$ by $20 \%$ ether-hexane.

By comparing their GLC $t_{R} s$ and GC/MS spectra with those of standards, peaks A, D, E and $F$ were identified as $\beta$-springene $\left(\mathrm{M}^{+} \mathrm{m} / z\right.$ 272), $n$-tricosane $\left(\mathrm{M}^{+} m / z\right.$ 324), $n$ pentacosane $\left(\mathrm{M}^{+} m / z 352\right)$ and $n$-heptacosane $\left(\mathrm{M}^{+} m / z\right.$ 380 ), respectively.

Peak B was concluded as the one-carbon-unit-larger homolog of $\beta$-springene, based on their similar GC/MS spectra (Figs. 1-1 and 1-2). The ${ }^{1} \mathrm{H}-\mathrm{NMR}$ spectrum (JEOL FX-100) of peaks A and B as a mixture (78:22) also supported the result and gave a characteristic methyl triplet $(\delta 0.96)$, assignable to the homoallyl methyl of peak B. In other words, the ethyl group was attached somewhere on the $\beta$-springene skeleton instead of the methyl groups and the triplet was not found in the spectrum of synthetic $\beta$-springene. The position of the ethyl branch was further assigned by GC/MS of per-hydrogenated peak $B$ as $C_{11}$ of $\beta$-springene, as indicated in Fig. 1-3. As a result, peak $B$ was concluded to be 11 -ethyl-7,15-dimethyl-

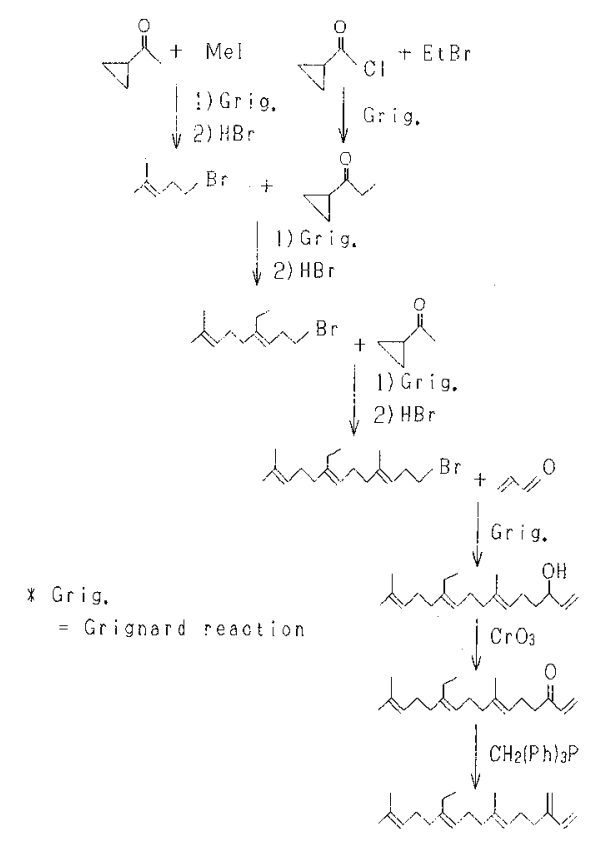

Scheme 1. Synthesis Route for Homo- $\beta$-springene.

3-methylene-hexadeca-1,6,10,14-tetraene.

A mixture of four possible isomers of the tetraene was synthesized by the method indicated (Scheme 1). ${ }^{5}$ One isomer corresponding to peak $\mathrm{B}$ was isolated from the mixture by preparative $\mathrm{AgNO}_{3}-\mathrm{SiO}_{2}-\mathrm{TLC}$ with $30 \%$ ether-hexane, and gave $\mathrm{M}^{+} 286.2661\left(\mathrm{C}_{21} \mathrm{H}_{34}\right.$, calc. 286.2659 ) by precise mass spectrometry. The ${ }^{1} \mathrm{H}-\mathrm{NMR}$ spectrum (Bruker $500 \mathrm{MHz}$ ) of the isomer gave the $\omega$ methyl triplet at $\delta 0.96$, while the original mixture of isomers gave two sets of the triplet at $\delta 0.98$ and $0.96 . Z$ and E-4-ethyl-8-methyl-1-bromo-nona-3,7-diene as standards indicated a similar triplet at $\delta 1.00$ and 0.95 , due to the double bond geometry at the 3 rd position. As a result, the $C_{10}$-geometry of the natural tetraene was suggested to be $E$. The other $\mathrm{C}_{6}$-geometry was the same as that of springene, and the chemical shift $(\delta 1.60)$ of the allyl methyl at $\mathrm{C}_{7}$ corresponded to the reported value ${ }^{6)}$ for $E$ $(\delta 1.59)$, while $Z$ gave it at $\delta 1.66$. As a result, the geometry of the two double bonds in natural tetraene were concluded to be $E, E$. Spectral data for the synthesized homo$\beta$-springene are as follows: ${ }^{1} \mathrm{H}-\mathrm{NMR}(500 \mathrm{MHz}): 6.38$ (q, 1), 4.95-5.30 (m, 7), 2.15-2.25 (br. m, 4), 1.95-2.10 (br. m, 10), $1.64(\mathrm{~s}, 3), 1.61$ (br. s, 6), $0.96(\mathrm{t}, 3)$.

Peak $C$ has not been assigned at present, but was suggested to be an hydrated homo- $\beta$-springene because of its polar nature and its similar GC/MS spectrum to that of homo- $\beta$-springene with the largest fragment at $m / z 286$, which corresponded to $\mathrm{M}^{+}-18$ of the possible alcohol.

This is the first case of homo- $\beta$-springene being iden-

* To whom all correspondence should be addressed. 

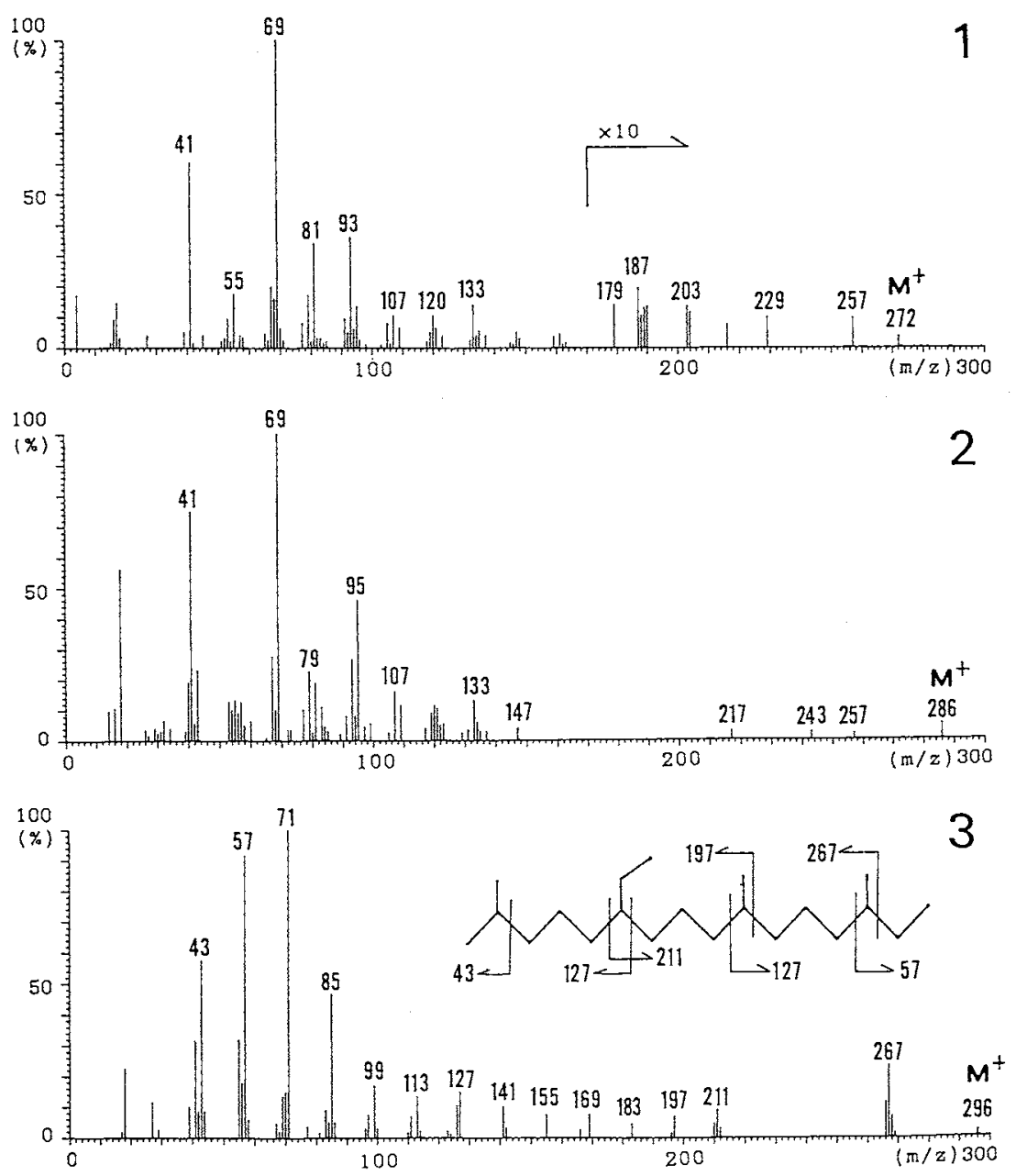

Fig. 1. GC/MS Spectra of the Sting Gland Components (Hitachi M-80B GC/MS, Operated at $70 \mathrm{eV}$ ). 1, peak A; 2, peak B; 3, per-hydrogenated peak B.

tified as a natural product, although $\beta$-springene is present in the dorsal gland secretion of the springbok, ${ }^{7}$ and also in the tobacco leaf. ${ }^{8)}$ Further studies on the biological functions of these compounds will be discussed elsewhere.

\section{References}

1) D. W. Hagstrum and B. J. Smittle, Environ. Entomol., 7, 594 (1978).

2) J. B. Waller, J. Insect Physiol, 11, 1595 (1965).
3) T. Piek, J. Insect Physiol., 12, 561 (1966).

4) H. S. Soliman, Bull. Soc. Fouad I Ent., 25, 1 (1941).

5) M. Julia, S. Julia and R. Guegan, Bull. Chim. Fr., 1960, 1072.

6) R. B. Bates and D. M. Gale, J. Am. Chem. Soc., 82, 5749 (1960).

7) B. V. Burger, M. L. Roux, H. S. C. Spies and V. Truter, Tetrahedron Lett., 52, 5221 (1978).

8) R. Uegaki, T. Fujimori, H. Kaneko and K. Kato, Agric. Biol. Chem., 44, 2215 (1980). 